\title{
Transplantations intracérébrales : attention! Une greffe peut en cacher une autre
}

\begin{abstract}
Dans un récent numéro de médecine/sciences étaient rapportés les premiers succès thérapeutiques de greffes intracérébrales de tissu médullo-surrénalien dans deux cas de maladie de Parkinson. Depuis la publication de ces deux premiers cas par les neurochirurgiens de Mexico [1], c'est plus d'une trentaine d'autres malades greffés dont les cas ont été présentés et c'est sans doute plus d'une centaine de malades qui ont déjà bénéficié de ce traitement dans des pays aussi divers que le Mexique, la Chine populaire, les ÉtatsUnis, Cuba ou le Brésil.
\end{abstract}

Toutes ces tentatives s'appuient sur des prémices théoriques qui semblaient claires : d'une part, la maladie de Parkinson est liée à la dégénérescence des neurones dopaminergiques situés dans le locus niger ; ces neurones innervent massivement le striatum, et c'est le déficit en dopamine au niveau de cette structure cible qui provoque les symptômes caractéristiques de la maladie ; d'autre part, la glande médullo-surrénale contient des cellules chromaffines qui produisent des catécholamines en grande quantité, substances qu'elles libèrent normalement dans la circulation. Les cellules chromaffines implantées dans le striatum pourraient donc suppléer les cellules de la substance noire en libérant au niveau des neurones cibles les neurotransmetteurs manquants. Cette hypothèse avait reçu dès 1983 un début d'application clinique en Suède [2], et la multiplication récente des implantations réussies tendrait à démontrer que ces prémices étaient
Un travail publié cet été dans Science [3] remet pourtant en cause simultanément l'explication des effets des greffes intracérébrales de tissu médullo-surrénalien et, peut-être, à terme, leur utilité thérapeutique. Dans ce travail, le MPTP (1-méthyl-4-phényl-1,2,5, 6-tétrahydropyridine), neurotoxine atteignant sélectivement les neurones catécholaminergiques et plus particulièrement dopaminergiques, a été injecté chez des souris. Dans cette espèce, le MPTP provoque une déplétion nette en dopamine dans le striatum, liée à une importante diminution de l'innervation dopaminergique; en revanche, et à l'inverse de ce qui se passe chez le primate ou chez l'homme, de nombreux neurones dopaminergiques survivent dans la substance noire. Une semaine après l'injection, des pièces de tissu provenant de la médullosurrénale d'autres souris adultes ont été implantées unilatéralement dans le striatum des souris traitées au MPTP. La présence d'une innervation dopaminergique a été jugée 2 à 6 semaines plus tard à l'aide d'un marquage immunocytochimique utilisant un anticorps reconnaissant la tyrosine hydroxylase, enzyme de synthèse des catécholamines. La surprise est venue de la mise en évidence d'une riche innervation dopaminergique unilatérale dans le striatum ayant reçu la greffe, le striatum opposé restant pratiquement déafférenté. Cette innervation ne peut provenir des cellules chromaffines implantées qui, à priori, ne peuvent se comporter comme des neurones du système nerveux central et qui, d'autre part étaient parfois très peu nombreuses à survivre dans les greffons. La conclusion des auteurs est donc que les greffes de tissu médullosurrénalien ont, dans ce cas, non pas suppléé les cellules de la substance noire, mais facilité la réinnervation du striatum à partir des cellules dopaminergiques survivantes chez l'hôte. Il s'agirait alors d'une action " trophique " et donc peut-être non spécifique du tissu médullo-surrénalien.

De cette conclusion à l'hypothèse selon laquelle des greffes intracérébrales de tissu surrénalien agiraient chez les malades parkinsoniens grâce à un renforcement de l'innervation dopaminergique de l'hôte et non pas à cause de la suppléance du système déficient, il n'y a qu'un pas que les auteurs ne font cependant qu'esquisser. Cette hypothèse, si elle se vérifiait, aurait des conséquences non négligeables sur les possibilités de succès des greffes à visée thérapeutique réalisées chez les malades parkinsoniens [4]. De telles greffes ne pourraient notamment avoir d'effet bénéfique qu'à condition que la destruction pathologique de l'innervation dopaminergique striatale ne soit pas complète. Comme l'évolution de la maladie de Parkinson se fait progressivement vers une telle destruction complète, on pourrait alors prévoir que l'effet des greffes s'atténuerait au cours du temps.

La démonstration d'un éventuel effet trophique des greffes de tissu médullo-surrénalien n'est cependant pas un argument définitif contre une véritable suppléance du système dopaminergique par 
les cellules chromaffines. Les expériences rapportées sur les sourisMPTP ne permettent pas, en particulier, d'apprécier un éventuel effet comportemental des greffes. Un des effets les plus frappants des greffes intracérébrales de tissu surrénalien chez l'homme est, par ailleurs, la bilatéralité des effets thérapeutiques d'une greffe pourtant unilatérale. Chez les souris MPTP, la réinnervation dopaminergique striatale est au contraire strictement unilatérale. Là encore, il est donc difficile de définir dans quelle mesure les résultats obtenus chez les souris-MPTP peuvent s'appliquer à l'homme. Il n'est pas impossible d'imaginer, en fait, que les deux effets rapportés des greffes intracébrales de tissu surrénalien (effet trophique et suppléance) pourraient coexister puisqu'il est établi [5] que les cellules chromaffines greffées libèrent de la dopamine dans le liquide céphalorachidien des malades greffés. L'article portant sur les souris
MPTP, en ne révélant l'effet trophique des greffes surrénaliennes qu'aujourd'hui, soit près de cinq ans après la première greffe chez l'homme, souligne un des aspects les plus curieux de cette aventure scientifique et médicale : le retard des données expérimentales [6]. Alors que l'on a produit au cours des dix dernières années des centaines d'articles concernant les greffes de neurones embryonnaires (dont les premières applications cliniques ne devraient voir le jour qu'au cours des mois à venir), on ne compte en effet qu'une à deux dizaines de travaux s'intéressant à des titres divers aux greffes intracérébrales de cellules de la glande médullosurrénale. Un vide que les neurobiologistes s'appliqueront sans doute à combler pour résoudre les problèmes théoriques posés par les données expérimentales obtenues... chez l'homme!

\section{RÉFÉRENCES}

1. Madrazo I, Drucker-Colin R, Diaz V, el al. Open microsurgical autograft of adrenal medulla to the right caudate nucleus in two patients with intractable Parkinson's disease. $N$ Engl J Med 1987; $316: 831-4$.

2. Backlund EO, Grandberg PO, Hamberger $\mathrm{B}$, et al. Transplantation of adrenal medullary tissue to striatum in parkinsonism : first clinical trials. $J$ Neurosurg $1985 ; 62$ : 169-73.

3. Bohn MC, Cupit L, Marciano F, Gash D. Adrenal medulla grafts enhance recovery of striatal dopaminergic fibers. Science 1987 ; 238 : 913-6.

4. More RY. Parkinson's disease : a new therapy? $N$ Engl J Med 1987; $316: 872-3$.

5. Freed WJ, Karoum F, Spoor HE, et al. Catecholamine content of intracerebral adre nal medulla grafts. Brain Res $1983 ; 269$ : 184-9.

6. Sladek JR (reported in Lewin R). Dramatic results with brain grafts. Science $1987 ; 237$ 245-7. 